\title{
ANAESTHETIC MANAGEMENT AND COMPLICATIONS OF CANINE ADRENALECTOMIES: 41 CASES (2007-2017)
}

\author{
Tristan MERLIN $^{1 *}$ and Kata VERES-NYÉKI ${ }^{2}$ \\ ${ }^{1}$ Eastcott Referrals, Edison Park, Hindle Way, Swindon, Wiltshire, SN3 3FR, \\ United Kingdom; ${ }^{2}$ Queen Mother Hospital for Animals, Royal Veterinary College, \\ Hatfield, United Kingdom
}

(Received 7 February 2019; accepted 2 May 2019)

\begin{abstract}
Little information is available about the management of adrenalectomies in veterinary anaesthesia. The aim of this study was to describe the anaesthetic techniques, the complication rate and outcome of these cases. Data were collected retrospectively from patients' records. Descriptive statistics were performed with Student's $t$-tests and Chi-square tests where appropriate. Forty-one cases were included. The mean age was $124.7 \pm 29.4$ months and the median body weight was $23.1 \mathrm{~kg}(5.3-69 \mathrm{~kg})$. Carcinoma was the most common diagnosis (34.1\%). Premedication was most commonly achieved with methadone alone (70.7\%) IV. Propofol was the most common induction agent (39\%). An infusion of opioids $(80.4 \%)$ and an epidural injection of morphine $(70.7 \%)$ were the most common analgesic techniques. Hypotension was the most common complication observed $(51.2 \%)$. The mortality rate was $14.6 \%$. The lowest recorded oesophageal temperature was statistically associated with the incidence of haemorrhage $(\mathrm{P}=0.025)$. The invasion of the vena cava $(\mathrm{P}=0.001)$ was significantly associated with haemorrhagic complications. The survival rate was better when patients received hydrocortisone intra-operatively $(\mathrm{P}=0.026)$. This study highlights some possible association between the anaesthetic management and the outcome of the procedure.
\end{abstract}

Key words: Anaesthesia, canine, adrenalectomies, complications, hydrocortisone

Primary adrenal gland tumours are rare in $\operatorname{dogs}(0.17 \%$ to $0.76 \%)$ (Massari et al., 2011) and surgical resection can be challenging. The perioperative complication rate can be as high as $51 \%$ in some studies (Kyles et al., 2003) with the mortality rate ranging between $13.5 \%$ and $60 \%$, the latter when including cases euthanised intra-operatively (Scavelli et al., 1986; Massari et al., 2011). These mortality rates have been improved following the introduction of new protocols for pre- and perioperative management. For example, the use of phenoxybenzamine preoperatively has been shown to decrease mortality from 48 to $13 \%$

*Corresponding author; E-mail: tmerlin@rvc.ac.uk; Phone: 0044 (01) 793-528341 
(Herrera et al., 2008) when given for a median of 20 days prior to the removal of a phaeochromocytoma.

Despite several publications on this subject, little attention has been given to the anaesthetic and analgesic management of those cases in veterinary medicine. Detailed descriptions of anaesthetic and analgesic protocols or the treatment of intra-operative complications are lacking in the majority of adrenalectomy studies. To our knowledge, there has been no evaluation of different anaesthetic or analgesic protocols with regards to their efficacy or the incidence of complications or survival. Thus, the objective of this study was to evaluate the anaesthetic management of adrenalectomies at a single academic centre over ten years.

The primary aim was to describe the anaesthetic and analgesic protocols, the encountered complications rate and the outcome. The secondary aim was to evaluate associations between preoperative conditions, anaesthetic or analgesic drugs and complications during the surgery or outcome.

\section{Materials and methods}

\section{Case collection}

An electronic database of all surgical cases admitted to the Royal Veterinary College was searched with the key word 'adrenal' to find potential candidates for inclusion in the study. The database was available between October 2007 and June 2017. Cases were selected if a unilateral or bilateral adrenalectomy was performed. Medical records were consulted to find the relevant medical history, demographic information, and the anaesthetic record of the procedure. Cases were excluded if the anaesthetic record was missing or incomplete with regards to the anaesthetic protocol used.

Ethics approval was given by the Clinical Research Ethical Review Board under the number 2017 1692-1.

Data collected were breed, age in months, sex, weight, side of the tumour, invasion of the vena cava, drugs used for premedication, induction, analgesia and maintenance of anaesthesia, surgical time, anaesthetic time, use of vasopressors or positive inotropes, use of phentolamine, use of hydrocortisone, use of preoperative phenoxybenzamine, histological diagnosis, cardiovascular parameters (heart rate, arterial blood pressure), lowest oesophageal temperature, and complications under general anaesthesia. The complications recorded were defined as follows:

- Arrhythmia: any deviation from sinus rhythm mentioned on the anaesthetic record.

- Haemorrhage: any blood loss mentioned on the anaesthetic record.

- Hypertension: any measurement of the systolic blood pressure (SBP) above $180 \mathrm{mmHg}$ when measured via an arterial catheter (invasive blood 
pressure, IBP) or a Doppler or any measurement of the mean blood pressure (MBP) above $120 \mathrm{mmHg}$ when measured with an oscillometric technique.

- Hypotension: any measurement of MBP lower than $60 \mathrm{mmHg}$ for more than $5 \mathrm{~min}$ if IBP or an oscillometric technique was used or SBP lower than $80 \mathrm{mmHg}$ if a Doppler was used.

- Death: any death or euthanasia occurring under anaesthesia or postoperatively within the first $72 \mathrm{~h}$ due to deteriorating clinical conditions and lack of improvement.

Continuous data were tested for normality with a Shapiro-Wilk test. Normally distributed variables were described with their mean and standard deviation (SD). Median, minimum and maximum values were used for non-normally distributed data. Qualitative data were described with frequencies and percentages. Comparison of continuous variables between two groups was performed with Student $t$-tests for normally distributed data or Mann-Whitney U-tests for nonnormally distributed data. Comparison of qualitative data was performed with Chisquare tests or Fisher exact tests where applicable. For variables that displayed more than two modalities (e.g., histological diagnosis) and for which the number of observations per modality was low, some of those modalities were grouped in order to perform comparisons between two groups only (e.g., phaeochromocytomas vs. non-phaeochromocytomas). Cases where the data relevant to the comparison performed was missing or unknown were not included. The level of significance was set at $5 \%(\mathrm{P}<0.05)$.

\section{Results}

Between November 2007 and June 2017, 57 cases of adrenalectomies were identified. These included four cats that were not included in the study. After application of inclusion and exclusion criteria, 41 cases were kept for analysis. All surgeries were performed by board-certified surgeons or supervised residents. All cases were anaesthetised by supervised anaesthesia residents or boardcertified anaesthetists.

There were 22 females $(22 / 41,53.6 \%)$ and 19 males (19/41, 46.3\%). Breeds included crossbreed (6 cases), Labrador, Lurcher (3 cases each), Boxer, Yorkshire Terrier, West Highland White Terrier, Staffordshire Bull Terrier, Shih Tzu (2 cases each), and 19 other different breeds for each remaining individual.

Age, surgery time and lowest recorded oesophageal temperature were normally distributed. Body weight and anaesthetic time were not normally distributed $(\mathrm{P}<0.05)$. Mean $\pm \mathrm{SD}$ age was $124.7 \pm 29.4$ months, mean $\pm \mathrm{SD}$ surgical time was $153.3 \pm 13.6$ min and mean \pm SD lowest recorded oesophageal temperature was $35 \pm 1.2^{\circ} \mathrm{C}$. 
Median (minimum-maximum) anaesthetic time was $235 \min (165-470 \mathrm{~min})$ and median (minimum-maximum) body weight was $23.1 \mathrm{~kg}(5.3-69 \mathrm{~kg})$.

Based on preoperative imaging (abdominal ultrasound and/or computed tomography), an adrenal mass or enlargement was present on the right side in $16 / 41$ cases $(39 \%)$, on the left side in $24 / 41$ cases $(58.5 \%)$ and was bilateral in $1 / 41$ cases $(2.4 \%)$. The vena cava was invaded in $11 / 41$ cases $(26.8 \%)$. The type of tumour invading the vena cava was a phaeochromocytoma in 8/11 cases and a carcinoma in $3 / 11$ cases.

Pre-operative results of investigations regarding the nature of the adrenal mass were available for twenty cases (20/41, 48.8\%). Fourteen cases of adrenaldependent hyperadrenocorticism were confirmed (14/20) along 6 phaeochromocytomas (6/20). For all remaining cases but five, a definite diagnosis was obtained after histological analysis (Table 1). For those five cases, the histological report was non-conclusive regarding the nature of the neoplasia.

\section{Table 1}

Postoperative histological diagnosis

\begin{tabular}{lcc}
\hline Histological type & Frequency & Percentage \\
\hline Adrenal cortical carcinoma & 14 & 34.1 \\
Phaeochromocytoma & 12 & 29.3 \\
Adrenal cortical adenoma & 7 & 17.1 \\
Unknown & 5 & 12.2 \\
Adrenal cortical hyperplasia & 1 & 2.4 \\
Suspected phaeochromocytoma & 1 & 2.4 \\
Haemangiosarcoma & 1 & 2.4 \\
\hline
\end{tabular}

All cases were monitored with the same standard equipment: 3-lead electrocardiograph (ECG), capnograph, pulse oximeter, blood pressure monitor (IBP, Doppler or oscillometric), oesophageal thermometer, anaesthetic agent and gas analyser. All animals but two had an arterial catheter placed in a dorso-pedal artery and 36/41 had a central venous catheter inserted in one of the two jugular veins.

\section{Anaesthetic protocol}

Premedication. All cases received an opioid, sometimes combined with acepromazine $(6 / 41,14.6 \%$, dose range: $0.005-0.02 \mathrm{mg} / \mathrm{kg})$, medetomidine $(3 / 41$, $7.3 \%, 0.005 \mathrm{mg} / \mathrm{kg}$ intramuscularly) or alfaxalone $(1 / 41,2.4 \%, 0.75 \mathrm{mg} / \mathrm{kg}$ intramuscularly). The opioid used most commonly was methadone $(39 / 41,95.1 \%$, dose range: $0.1-0.4 \mathrm{mg} / \mathrm{kg}$, intravenously or intramuscularly), used as the sole premedication agent in 29/41 cases $(70.7 \%)$. Other opioids used were morphine or pethidine in one case each $(1 / 41,2.4 \%)$. Table 2 summarises the different combinations used for premedication. 
Table 2

Drug combinations used for premedication

\begin{tabular}{lcc}
\hline Drug combination & Frequency & Percentage \\
\hline $\begin{array}{l}\text { Methadone alone } \\
(0.1 \mathrm{mg} / \mathrm{kg} \text { intramuscularly }-0.4 \mathrm{mg} / \mathrm{kg} \text { intramuscularly) }\end{array}$ & 29 & 70.7 \\
$\begin{array}{l}\text { Acepromazine } \\
(0.005-0.02 \mathrm{mg} / \mathrm{kg})+\text { Methadone }(0.2-0.3 \mathrm{mg} / \mathrm{kg})\end{array}$ & 6 & 14.6 \\
$\begin{array}{l}\text { Medetomidine } \\
(0.001 \text { intravenously }-0.005 \text { intramuscularly } \mathrm{mg} / \mathrm{kg})+\end{array}$ & 3 & 7.3 \\
Methadone $(0.2-0.3 \mathrm{mg} / \mathrm{kg}$ intramuscularly $)$ & & \\
$\begin{array}{l}\text { Alfaxalone } \\
(0.75 \mathrm{mg} / \mathrm{kg} \text { intramuscularly })+\end{array}$ & 1 & 2.4 \\
Methadone $(0.3 \mathrm{mg} / \mathrm{kg}$ intramuscularly $)$ & 1 & 2.4 \\
Morphine $(0.2 \mathrm{mg} / \mathrm{kg}$ intravenously $)$ & 1 & 2.4 \\
Pethidine & &
\end{tabular}

Induction. The most commonly used agent for induction of anaesthesia was propofol (30/41, 73.1\%) either alone or combined to a benzodiazepine (14/30). Alfaxalone was used in six cases $(6 / 41,14.6 \%)$. Benzodiazepines were used for 23 cases in total and were either diazepam in three cases (3/23) or midazolam in 20 cases (20/23). Fentanyl was used as an induction agent in 3/41 cases $(7.3 \%)$ always in combination with midazolam. Propofol and midazolam were combined with lidocaine (1-2 mg/kg intravenously) in two cases. Other combinations and drugs are reported in Table 3.

\section{Table 3}

Drug combinations used for induction

\begin{tabular}{lcc}
\hline Drug combination & Frequency & Percentage \\
\hline Propofol alone & 16 & 39 \\
Propofol + Midazolam $(0.2-0.5 \mathrm{mg} / \mathrm{kg})$ & 9 & 21.9 \\
Alfaxalone + Midazolam $(0.3-0.4 \mathrm{mg} / \mathrm{kg})$ & 4 & 9.8 \\
Fentanyl & 3 & 7.3 \\
$(0.005-0.01 \mathrm{mg} / \mathrm{kg}$ intravenously $)+$ Midazolam $(0.2-0.5 \mathrm{mg} / \mathrm{kg})$ & 3 & 7.3 \\
Propofol + Diazepam $(0.2-0.4 \mathrm{mg} / \mathrm{kg})$ & 2 & 4.8 \\
Alfaxalone & 2 & 4.8 \\
Etomidate + Midazolam & 2 & 4.8 \\
Propofol + Midazolam $(0.2-0.5 \mathrm{mg} / \mathrm{kg})+$ Lidocaine $(1-2 \mathrm{mg} / \mathrm{kg})$ & 2 & \\
\hline
\end{tabular}


Maintenance. Isoflurane was used in 27/41 (65.9\%) cases and sevoflurane in $14 / 41(34.1 \%)$ cases.

\section{Analgesic protocol}

Table 4 summarises the different drugs used for analgesia and Table 5 their combinations. The opioid used in the CRI was fentanyl (dose range: $0.05-$ $0.4 \mu \mathrm{g} / \mathrm{kg} / \mathrm{min}$ ) in 28 cases $(28 / 41,68.3 \%$ ), remifentanil (dose range: $0.1-0.3 \mu \mathrm{g} /$ $\mathrm{kg} / \mathrm{min})$ in four cases $(4 / 41,9.8 \%)$ and morphine $(0.1 \mathrm{mg} / \mathrm{kg} / \mathrm{h})$ in one case.

Table 4

Drugs used for analgesia. Total number of cases and sum of percentages are above $100 \%$ due to combinations (see Table 5)

\begin{tabular}{lcc}
\hline Analgesic drug and dose range when available & Frequency & Percentage \\
\hline Constant Rate Infusion (CRI) of opioids & 33 & 80.4 \\
\hline Morphine epidural $(0.1-0.2 \mathrm{mg} / \mathrm{kg})$ & 29 & 70.7 \\
\hline Lidocaine CRI (30-60 $\mu \mathrm{g} / \mathrm{kg} / \mathrm{min})$ & 7 & 17.1 \\
\hline $\begin{array}{l}\text { Ropivacaine epidural }(1 \mathrm{mg} / \mathrm{kg} \text { in } 0.2 \mathrm{~mL} / \mathrm{kg} ; \\
0.5 \mathrm{mg} / \mathrm{kg} \text { in } 0.3 \mathrm{~mL} / \mathrm{kg} \text {; high epidural L1-L2 with } 0.1 \mathrm{~mL} / \mathrm{kg})\end{array}$ & 3 & 7.3 \\
\hline $\begin{array}{l}\text { Transverse abdominis plane (TAP) block } \\
\text { (maximum dose } 2 \mathrm{mg} / \mathrm{kg} \text { diluted) }\end{array}$ & 3 & 7.3 \\
\hline $\begin{array}{l}\text { Local instillation of ropivacaine } \\
\text { (maximum dose calculated } 1.5 \mathrm{mg} / \mathrm{kg})\end{array}$ & 2 & 4.9 \\
\hline Ultrasound-guided paravertebral block & 2 & 4.9 \\
\hline Ketamine (0.5 mg/kg single injection) & 1 & 2.4 \\
\hline Nitrous oxide (inspired fraction: $50-66 \%)$ & 1 & 2.4 \\
\hline
\end{tabular}

\section{Other perioperative drugs}

As part of the preoperative management of suspected phaeochromocytomas, $17 / 41$ dogs received phenoxybenzamine prior to surgery $(0.3-0.9 \mathrm{mg} / \mathrm{kg}$; frequency of administration and duration of treatment prior to surgery not available). All twelve cases of phaeochromocytomas were treated preoperatively with phenoxybenzamine and in six cases this treatment was initiated following a preoperative diagnosis. The remaining six phaeochromocytomas and the five other cases that received phenoxybenzamine prior to the surgery were pre-treated on the basis of a clinical suspicion.

Patients who had a confirmed diagnosis of hyperadrenocorticism prior to the surgery (14 cases) were treated with trilostane in five cases (dose and duration of the treatment not available). All patients who had a hyperadrenocorticism 
confirmed prior to the surgery received a constant rate infusion of hydrocortisone under general anaesthesia $(0.5 \mathrm{mg} / \mathrm{kg}$ /hour started $1 \mathrm{~h}$ before induction and continued 12 to $24 \mathrm{~h}$ postoperatively). Some cases received hydrocortisone in the absence of pre-operative hyperadrenocorticism (including six undiagnosed phaeochromocytomas). In total 24/41 cases received a hydrocortisone CRI and one dog received an infusion of dexamethasone over $4 \mathrm{~h}$ (total dose administered $0.5 \mathrm{mg} / \mathrm{kg}$ ) under general anaesthesia.

Table 5

Drug combinations used for analgesia

\begin{tabular}{lcc}
\hline Analgesic combination & Frequency & Percentage \\
\hline Fentanyl CRI + Morphine epidural & 14 & 34.1 \\
\hline Fentanyl CRI & 5 & 12.2 \\
\hline Methadone (premedication) + Morphine epidural + & 3 & 7.3 \\
\hline Ropivacaine epidural & 3 & 7.3 \\
\hline Remifentanil CRI + Morphine epidural & 2 & 4.9 \\
\hline Fentanyl CRI + Lidocaine CRI & 2 & 4.9 \\
\hline Fentanyl CRI + Lidocaine CRI + Morphine epidural & 2 & 4.9 \\
\hline Fentanyl CRI + Morphine epidural + Local ropivacaine & 1 & 2.4 \\
\hline Fentanyl CRI + Lidocaine CRI + Morphine epidural + & 1 & 2.4 \\
\hline Nitrous oxide & 1 & 2.4 \\
\hline Fentanyl CRI + Morphine epidural + TAP block & 1 & 2.4 \\
\hline Fentanyl CRI + TAP block & 1 & 2.4 \\
\hline Methadone (premedication + bolus) + Lidocaine CRI & 1 & 2.4 \\
\hline Methadone (premedication) + Fentanyl boluses + & 1 & 2.4 \\
\hline Paravertebral block & 1 & 2.4 \\
\hline Methadone (premedication) + Paravertebral block (T10-T11) & 1 & 2.4 \\
\hline Morphine epidural + Lidocaine CRI & 1 & 2 \\
\hline Morphine epidural + Morphine CRI + Ketamine & 1 & \\
\hline Morphine epidural + TAP block & 1 & 2 \\
\hline Remifentanil CRI & 1 & \\
\hline
\end{tabular}

For cases with an invasion of the vena cava by a thrombus or an extension of the tumour, a venotomy was performed (11/41, 26.8\%). Unfractionated heparin was given intravenously (30-50 IU/kg) either before or after the venotomy was performed to $8 / 11$ dogs (two carcinomas and six phaeochromocytomas). 


\section{Complications}

In 30/41 cases (73.1\%) at least one perioperative complication was seen. Table 6 summarises the different combinations of complications encountered.

Hypotension was the most common complication observed (21/41, 51.2\%) and treated at the discretion of the attending anaesthetist. Crystalloid boluses were administered in 12/41 cases (29.3\%) and colloid boluses in 13/41 cases (31.7\%, either a gelatine-based or a starch-derived colloid depending on the available product).

Table 6

Combinations of complications under general anaesthesia

\begin{tabular}{lcc}
\hline Complications & Frequency & Percentage \\
\hline Hypertension alone & 6 & 20 \\
Hypotension alone & 6 & 20 \\
Hypotension + Haemorrhage & 4 & 13.3 \\
Hypotension + Arrhythmia & 3 & 10 \\
Hypotension + Hypertension & 3 & 10 \\
Hypotension + Hypertension + Haemorrhage & 3 & 10 \\
Arrhythmia + Hypertension & 2 & 6.6 \\
Hypotension + Hypertension + Haemorrhage + Arrhythmia & 2 & 6.6 \\
Haemorrhage alone & 1 & 3.3
\end{tabular}

Bradycardia was treated with atropine (10-20 $\mu \mathrm{g} / \mathrm{kg}$ intravenously) or glycopyrrolate $(5-10 \mu \mathrm{g} / \mathrm{kg}$ intravenously) in 13 cases $(13 / 41,31.7 \%)$.

The use of a vasopressor or positive inotrope was recorded in 13/41 cases (31.7\%). Drugs used were either dopamine (5-10 $\mu \mathrm{g} / \mathrm{kg} / \mathrm{min}$ intravenously, $9 / 41$ cases, $21.9 \%$ ), noradrenaline (0.1-0.2 $\mu \mathrm{g} / \mathrm{kg} / \mathrm{min}$ intravenously, $2 / 41$ cases, $4.9 \%$ ), phenylephrine as single bolus ( $3 \mu \mathrm{g} / \mathrm{kg}$ intravenously, $2 / 41$ cases, $4.9 \%)$ or as a CRI $(1-2 \mu \mathrm{g} / \mathrm{kg} / \mathrm{min}$ intravenously, $1 / 41$ case, $2.4 \%)$ or ephedrine $(0.05 \mathrm{mg} / \mathrm{kg}$ intravenously, $4 / 41,9.8 \%)$. A combination of those options was used in five cases $(5 / 41,12.2 \%)$.

Hypertension was reported in 16 cases $(16 / 41,39 \%)$. The exact maximum SBP was not always available for analysis as some records only mention spikes in HR and BP (above $180 \mathrm{mmHg}$ ). When recorded, maximum SBP at the time of the hypertensive episode ranged between 190 and $240 \mathrm{mmHg}$. In the subgroup of phaeochromocytomas, 7/12 cases had a hypertensive episode.

Phentolamine $(0.5-3 \mu \mathrm{g} / \mathrm{kg} / \mathrm{min} \pm 0.2-0.5 \mathrm{mg} / \mathrm{kg}$ bolus intravenously) was used in $7 / 41$ cases $(17.1 \%)$. Six of those seven cases were confirmed or suspected phaeochromocytomas and one was a carcinoma.

Haemorrhage was reported in 10 cases $(10 / 41,24.4 \%)$. In total, 5/41 cases $(12.2 \%)$ received a blood transfusion $(10 \mathrm{~mL} / \mathrm{kg})$. 
In one case, the transfusion was started due to an acute haemoabdomen which was diagnosed prior to the surgery and the volume lost could not be calculated. In the other four cases, the transfusion was started due to blood losses during the surgery, although only one case had a record of the amount of blood lost ( $40 \%$ estimated at the end of the surgery). In the remaining six cases of haemorrhage, the volume lost was estimated between $7.6 \%$ and $40 \%$ of total blood volume.

Different types of arrhythmias were reported in seven dogs (7/41, 17.1\%). Ventricular tachycardia and sporadic ventricular premature complexes were reported in three dogs (in one case those arrhythmias were present before induction). Two dogs had some episodes of supraventricular tachycardia or atrial premature complexes. Finally, one dog had an atrial standstill following the first surgical incision and in one dog a temporary sinus arrest was observed followed later during the procedure by a temporary depression of the ST segment. This last case was receiving a lidocaine infusion when the arrhythmia occurred and was one of the two cases of phaeochromocytomas to display some arrhythmic features. The atrial standstill responded to the initiation of a phentolamine CRI. As they were only temporary and self-resolving, no treatment was recorded in the other cases. In four of those seven cases, a history of preoperative arrhythmias was reported.

\section{Outcome}

Overall, six deaths were recorded during the first three days after the surgery (mortality rate 6/41,14.6\%; three phaeochromocytomas, two carcinomas and one haemangiosarcoma). Four of those cases were euthanised, one died during the recovery phase and the last one suffered from cardiopulmonary arrest three days after the surgery. Only this last animal underwent necropsy but no obvious cause for the death was identified (suspected cortical carcinoma, no invasion of the vena cava, preoperative history of ventricular arrhythmias and elevated cardiac troponins).

In three cases (all euthanised between day 2 and day 3 after the surgery), animals displayed clinical signs that were deemed compatible with a pulmonary thromboembolism (acute onset of tachypnoea, low peripheral haemoglobin oxygen saturation, exclusion of all other possible causes for the observed clinical signs, predisposing factors). Two of those cases had a phaeochromocytoma and one a cortical carcinoma. Both phaeochromocytoma cases were receiving phenoxybenzamine before the surgery due to a high clinical suspicion in the absence of a definite diagnosis. All three cases had a venotomy performed and none of them received perioperative unfractionated heparin.

The fourth case euthanised was displaying signs of recurrent untreatable postsurgical bleeding probably due to a disseminated intravascular coagulopathy. This case had been admitted for a haemoabdomen and the adrenal mass removed was a metastasised haemangiosarcoma without any invasion of the vena cava. 
Finally, the dog that died during recovery had been admitted to surgery for the removal of an invading phaeochromocytoma and had been treated with phenoxybenzamine for 3 days. A venotomy was performed at a low core temperature (between 32 and $33{ }^{\circ} \mathrm{C}$ ) and while closing the abdominal cavity, the blood pressure decreased progressively without responding to any vasopressor administered until cardiac arrest occurred.

\section{Statistical analysis}

Continuous variables. The lowest recorded oesophageal temperature was statistically associated with the incidence of haemorrhage with a mean $( \pm \mathrm{SD})$ temperature for cases that experienced haemorrhage $(10 / 39-25.6 \%)$ of $34.3{ }^{\circ} \mathrm{C}$ \pm 0.91 versus $35.3{ }^{\circ} \mathrm{C} \pm 1.26$ for non-haemorrhagic patients $(29 / 39-74.4 \%)(\mathrm{P}=$ $0.025)$.

Categorical variables. When separating phaeochromocytomas from all other types of tumours, male dogs (regardless of their neutering status) were overrepresented amongst phaeochromocytoma cases $(9 / 13-69.2 \%)(\mathrm{P}=0.045)$. Phaeochromocytomas invaded the vena cava significantly more $(8 / 11$ cases $72.7 \%)$ than non-phaeochromocytomas $(\mathrm{P}=0.001)$.

Patients that did not receive hydrocortisone during the procedure (16 cases) are overrepresented amongst the non-survivors ( 5 cases $-83.3 \%$ of the total number of deceased cases) $(\mathrm{P}=0.026)$.

Three out the six cases $(50 \%)$ that received alfaxalone died compared to $3 / 35(8.5 \%)$ cases that did not receive alfaxalone $(\mathrm{P}=0.031)$.

\section{Discussion}

To the authors' knowledge, this is the first report about adrenalectomies primarily focusing on the anaesthetic and analgesic management over 10 years at a single institution.

In agreement with earlier publications, adrenalectomies were performed rarely during the study period, mostly on old dogs and there was no predisposition of age or breed (van Sluijs et al., 1995; Barthez et al., 1997; Anderson et al., 2001; Kyles et al., 2003; Schwartz et al., 2008; Jimenez Pelez et al., 2008; Herrera et al., 2008; Massari et al., 2011; Lang et al., 2011; Barrera et al., 2013; Naan et al., 2013) In the present study, the proportion of males affected by phaeochromocytomas was significantly higher, similarly to the findings of Kyles et al. (2003). Carcinoma was the predominant type of neoplasia followed by phaeochromocytomas. Phaeochromocytomas seemed to invade the vena cava more often than other tumours. There was no statistical association between the type of neoplasia, the surgical time, the anaesthetic time and the incidence of complications as reported before. (Schwartz et al., 2008; Massari et al., 2011; Lang et al., 2011) 
The mortality rate in this study $(14.6 \%)$ was higher than what was reported previously in the general canine population (between $0.17 \%$ and $1.35 \%$ ), even when considering only cases with a higher ASA status $(1.33 \%$ for cases with an ASA status of 3 to 5) (Brodbelt, 2009; Bille et al., 2011). Although half of the deaths were associated with a phaeochromocytoma, the mortality rate in the present study is consistent with the latest publications regarding adrenalectomies in general (Lang et al., 2011; Barrera et al., 2013; Naan et al., 2013). One of the main difficulties when comparing mortality rates is the consistency of the definition given to perioperative mortality. We chose to include cases that were euthanised due to the fact that euthanasia was elected because of a clinical deterioration, likely due to the underlying disease and recent surgery, that would have probably resulted in death eventually. It could have been interesting to separate the morbidity related to an emergency procedure from the morbidity related to elective cases. However, as we mentioned previously this information was not always available in the dataset and the limited number of deaths recorded would probably weaken any statistical association.

Another finding of this study was the association between the use of alfaxalone and overall death. Four cases that died in this case series were euthanised because of a deterioration in their clinical condition presumably because of pulmonary thromboembolisms or a disseminated intravascular coagulation and three of them received alfaxalone for induction. Although the overall number of cases induced with alfaxalone was limited to 6 and thus, the interpretation of this association must be very prudent, this finding is interesting and has never been reported previously to the authors' knowledge. However, correlation does not necessarily prove an underlying causal relationship and this finding must be reevaluated with a larger sample. Alfaxalone is a recent induction agent in the history of veterinary anaesthesia and is not in use in human medicine at the time of writing. No effect on the coagulation cascade has been identified as of yet but this may be a finding that could warrant further exploration.

The use of hydrocortisone was associated with increased survival. As reported, in our population hydrocortisone was systematically used for hyperadrenocorticism cases prior to the surgery due to the risk of postoperative adrenal insufficiency. This supplementation was inconsistent in all other cases. However, the nature of the tumour had not been determined in all cases prior to the surgical intervention. In some instances, potentially steroid-secreting epithelial tumours were identified on histopathological examination and would have benefited from perioperative steroid supplementation.

For non-steroid-producing tumours, the perioperative use of corticoids is currently poorly described in the literature. In critical illness or following major surgery, the endogenous secretion of cortisol will normally increase (Shaikh et al., 2012). However, it is difficult to determine pre-operatively whether this response will be appropriate in a given patient. Moreover, in human patients chron- 
ic secretion of catecholamines by phaeochromocytomas will tend to downregulate adrenergic receptors and removal of the affected gland will abruptly decrease the plasmatic levels of circulating catecholamines (Connor and Boumphrey, 2016). One of the effect of steroids (endogenous or exogenous) is to increase the sensitivity of those receptors to catecholamines (Shaikh et al., 2012).

Thus, we believe that the association found between the lack of perioperative supplementation with hydrocortisone and death could reflect an unexpected state of postoperative hypoadrenocorticism either due to an insufficient endogenous response to the surgical stress, a missed diagnosis of hyperadrenocorticism or because of a decreased response to and concentration of endogenous catecholamines when considering phaeochromocytomas.

Haemorrhage was also associated with a lower mean minimal oesophageal temperature. This may reflect a deliberate choice from the anaesthetist to achieve hypothermia in some cases, but this information was not recorded. Hypothermia has been shown to decrease oxygen consumption and minimise organ dysfunction in the event of low circulation or circulatory arrest, as what may happen during occlusion of the venous inflow (Brodeur et al., 2017). However, it may also affect the canine coagulation system and promote hypocoagulation, by inhibiting the enzymatic coagulation cascade, sequestration of platelets in the spleen and the liver and decreased platelet aggregation (Clark-Price, 2015; Sessler, 2016; Brodeur et al., 2017). In humans, hypothermia has also directly been associated with an increase of $16 \%$ in blood losses for each degree below the normal temperature (Rajagopalan et al., 2008). With regard to adrenalectomies, further prospective research should be performed to identify a potential benefit of therapeutic hypothermia when an occlusion of the vena cava needs to be performed.

In this study, the second most common analgesic treatment administered to the patients was an epidural injection of morphine (sometimes combined with a local anaesthetic drug). When morphine was used alone, a strong opioid CRI was always combined with the epidural, but on the three occasions when ropivacaine was injected with the epidural morphine no further opioid than the initial methadone for premedication was injected. The analgesic efficacy of preservative free morphine in the epidural space has been studied in dogs undergoing laparotomies and proved to be beneficial (Pekcan and Koc, 2010). In humans, there is some evidence that epidural anaesthesia could decrease hypercoagulation (Tuman et al., 1991; Williams et al., 1999; Hollmann, 2001) and improve mortality rates following major vascular surgeries but not necessarily improve morbidity. In the present study, we did not identify any correlation between the use of an epidural and the incidence of complications. Further prospective research on a large sample may be warranted to identify a benefit of epidurals on the canine coagulation system or in the setting of a major vascular surgery.

Although it is known that the lack of analgesia can affect the surgical prognosis by increasing the stress response, the oxygen consumption, the time to 
complete wound healing (Wiese and Yaksh, 2015), to date there is no report proving that one analgesic technique can affect significantly the prognosis of exploratory laparotomies in veterinary medicine. In the present study, no association between any analgesic drug or combination and one of the studied outcomes could be identified.

Due to the retrospective nature of the study, many different anaesthetic and analgesic protocols and combinations were described in this paper. This variety can be explained by the number of different anaesthetists in charge over the 10 years covered by the study, the availability of different drugs at a certain point in time and the description of newer techniques such as the paravertebral or the transverse abdominis plane block. It is almost impossible to justify retrospectively the use of one protocol over the other for each case and it is very likely that the choice made at the time was based on a combination of personal preference for one technique or protocol and the perceived need based on the clinical examination and planned surgical technique.

Due to the numerous combinations and parameters included in this study, it is possible that the use of repeated univariate tests has led to underpowered conclusions. A multivariate analysis would be the step to take if more cases would become available for analysis. As mentioned earlier many drugs or combinations were used on a small number of individuals and this could create an even bigger bias in a multivariate analysis. Therefore, this type of analysis was not performed but some significant findings may have been missed.

\section{Acknowledgements}

The authors would like to thank the theatre team for their record keeping of and for facilitating the access to the material used in this study, all the anaesthetists involved in the cases used, Mr David Brodbelt and Ms Yu-Mei Chang for the statistical advice.

\section{References}

Anderson, C. R., Birchard, S. J., Powers, B. E., Belandria, G. A., Kuntz, C. A. and Withrow, S. J. (2001): Surgical treatment of adrenocortical tumors: 21 cases (1990-1996). J. Am. Anim. Hosp. Assoc. 37, 93-97.

Barrera, J. S., Bernard, F., Ehrhart, E. J., Withrow, S. J. and Monnet, E. (2013): Evaluation of risk factors for outcome associated with adrenal gland tumors with or without invasion of the caudal vena cava and treated via adrenalectomy in dogs: 86 cases (1993-2009). J. Am. Vet. Med. Assoc. 242, 1715-1721.

Barthez, P. Y., Marks, S. L., Woo, J., Feldman, E. C. and Matteucci, M. (1997): Pheochromocytoma in dogs: 61 cases (1984-1995). J. Vet. Intern. Med. 11, 272-278.

Bille, C., Auvigne, V., Libermann, S., Bomassi, E., Durieux, P. and Rattez, E. (2011): Risk of anaesthetic mortality in dogs and cats: an observational cohort study of 3546 cases. Vet. Anaesth. Analg. 39, 59-68. 
Brodbelt, D. (2009): Perioperative mortality in small animal anaesthesia. Vet. J. 182, 152-161.

Brodeur, A., Wright, A. and Cortes, Y. (2017): Hypothermia and targeted temperature management in cats and dogs. J. Vet. Emerg. Crit. Car. 27, 151-163.

Clark-Price, S. (2015): Inadvertent perianesthetic hypothermia in small animal patients. Vet. Clin. North Am. Small Anim. Pract. 45, 983-994.

Connor, D. and Boumphrey, S. (2016): Perioperative care of phaeochromocytoma. BJA Educ. 16, $153-158$.

Herrera, M. A., Mehl, M. L., Kass, P. H., Pascoe, P. J., Feldman, E. C. and Nelson, R. W. (2008): Predictive factors and the effect of phenoxybenzamine on outcome in dogs undergoing adrenalectomy for pheochromocytoma. J. Vet. Intern. Med. 22, 1333-1339.

Hollmann, M. (2001): Epidural anesthesia prevents hypercoagulation in patients undergoing major orthopedic surgery. Reg. Anaesth. Pain Med. 26, 215-222.

Jimenez Pelez, M., Bouvy, B. M. and Dupré, G. P. (2008): Laparoscopic adrenalectomy for treatment of unilateral adrenocortical carcinomas: technique, complications, and results in seven dogs. Vet. Surg. 37, 444-453.

Kyles, A. E., Feldman, E. C., Cock, H. E. V. D., Kass, P. H., Mathews, K. G., Hardie, E. M., Nelson, R. W., Ilkiw, J. E. and Gregory, C. R. (2003): Surgical management of adrenal gland tumors with and without associated tumor thrombi in dogs: 40 cases (1994-2001). J. Am. Vet. Med. Assoc. 223, 654-662.

Lang, J. M., Schertel, E., Kennedy, S., Wilson, D., Barnhart, M. and Danielson, B. (2011): Elective and emergency surgical management of adrenal gland tumors: 60 cases (1999-2006). J. Am. Anim. Hosp. Assoc. 47, 428-435.

Massari, F., Nicoli, S., Romanelli, G., Buracco, P. and Zini, E. (2011): Adrenalectomy in dogs with adrenal gland tumors: 52 cases (2002-2008). J. Am. Vet. Med. Assoc. 239, 216-221.

Naan, E. C., Kirpensteijn, J., Dupré, G. P., Galac, S. and Radlinsky, M. G. (2013): Innovative approach to laparoscopic adrenalectomy for treatment of unilateral adrenal gland tumors in dogs. Vet. Surg. 42, 710-715.

Pekcan, Z. and Koc, B. (2010): The post-operative analgesic effects of epidurally administered morphine and transdermal fentanyl patch after ovariohysterectomy in dogs. Vet. Anaesth. Analg. 37, 557-565.

Rajagopalan, S., Mascha, E., Na, J. and Sessler, D. I. (2008): The effects of mild perioperative hypothermia on blood loss and transfusion requirement. Anesthesiology 108, 71-77.

Scavelli, T. D., Peterson, M. E. and Matthiesen, D. T. (1986): Results of surgical treatment for hyperadrenocorticism caused by adrenocortical neoplasia in the dog: 25 cases (1980-1984). J. Am. Vet. Med. Assoc. 189, 1360-1364.

Schwartz, P., Kovak, J. R., Koprowski, A., Ludwig, L. L., Monette, S. and Bergman, P. J. (2008): Evaluation of prognostic factors in the surgical treatment of adrenal gland tumors in dogs: 41 cases (1999-2005). J. Am. Vet. Med. Assoc. 232, 77-84.

Sessler, D. I. (2016): Perioperative thermoregulation and heat balance. Lancet 387, 2655-2664.

Shaikh, S., Verma, H., Yadav, N., Jauhari, M. and Bullangowda, J. (2012): Applications of steroid in clinical practice: A review. ISRN Anesthesiol. 2012, 1-11. Article ID 985495.

Tuman, K. J., McCarthy, R. J., March, R. J., Delaria, G. A., Patel, R. V. and Ivankovich, A. D. (1991): Effects of epidural anesthesia and analgesia on coagulation and outcome after major vascular surgery. Anaesth. Pain Intensive Care 73, 696-704.

Van Sluijs, F. J., Sjollema, B. E., Voorhout, G., Van Den Ingh, T. S. G. A. M. and Rijnberk, A. (1995): Results of adrenalectomy in 36 dogs with hyperadrenocorticism caused by adrenocortical tumour. Vet. Quart. 17, 113-116.

Wiese, A. J. and Yaksh, T. L. (2015): Nociception and pain mechanisms. In: Gaynor, J. S. and Muir, W. W. (eds) Handbook of Veterinary Pain Management. Elsevier, Mosby. pp. 10-41.

Williams, J. P., Sullivan, E. A. and Ramakrishna, H. (1999): Effects of thoracic epidural anaesthesia on the coagulation system. Best Pract. Res. Clin. An. 13, 31-56. 